\title{
Quantitative multi-elemental laser-induced breakdown spectroscopy using artificial neural networks
}

Vincent Motto-Ros

\author{
Alexander S. Koujelev \\ alexander.koujelev@space.gc.ca
}

Gordon R. Osinski

Alexander E. Dudelzak

\author{
Canadian Space Agency, 6767 Route de l’Aéroport, Longueuil, Québec, Canada J3Y 8Y9 \\ Canadian Space Agency, 6767 Route de l'Aéroport, Longueuil, Québec, Canada J3Y 8Y9 \\ Current address: Department of Earth Sciences/Department of Physics and Astronomy, University of \\ Western Ontario, 1151 Richmond Street, London, Ontario, N6A 5B7 Canada \\ Canadian Space Agency, 6767 Route de l’Aéroport, Longueuil, Québec, Canada J3Y 8Y9
}

The Laser-Induced Breakdown Spectroscopy (LIBS) is an emerging technique with great potential in atomic elemental analysis in many areas, particularly, in space exploration. In this paper, an algorithm for automated identification of elements and measurements of their concentrations in rocks and soils, as well as its experimental validation are presented. The proposed approach is based on the artificial neural network (ANN). We demonstrate that the ANN algorithm works successfully for all major elements of geological interest tested on natural rock and soil samples. [DOI: 10.2971/jeos.2008.08011]

Keywords: Laser-induced breakdown spectroscopy (LIBS), artificial neural network (ANN), quantitative spectroscopy of minerals, laser instrumentation for planetary exploration

\section{INTRODUCTION}

The Laser-Induced Breakdown Spectroscopy (LIBS) concept relies on the analysis of plasma created by focusing a laser beam onto a sample of interest, which can be in solid, liquid or gas phase. Spectral analysis of light emitted by the plasma allows determining the nature and the chemical composition of the material [1]. During the last decade, LIBS has emerged as a highly promising analytical technique. It has been proposed for multi-elemental analysis in many areas such as geology, metallurgy, pharmaceutical, homeland-security, and environmental applications [2]-[6]. In particular, LIBS has been considered a very promising technique for future planetary exploration-lander missions [7, 8]. LIBS offers greater capabilities as compared to the techniques used in the previous roverbased Martian missions such as X-ray fluorescence (XRF) or alpha-proton-X spectroscopy (APXS) $[9,10]$. LIBS does not require sample preparation and allows for depth profiling of weathered rocks; measurement can be done remotely and the results delivered in real or quasi-real time; the dust layers, normally an interfering factor in other techniques, do not impede LIBS. Due to these features LIBS instruments have been selected as payloads on the 2009 Mars Science Laboratory (MSL) and 2011 ExoMars missions [11, 12]. While the LIBS technology has been well developed and mature by now generally, two main issues remain being important research and development subjects - instrument miniaturization, and LIBS data interpretation including retrieving quantitative information from measured spectra. The latter is especially important for an exploration tool when the target properties and composition are a priori unknown [13]. Compositions of crustal materials such as rocks and dust are usually very complex with a large scale of concentration levels. This, along with the socalled matrix effects [14], may result in significant overlapping of spectral lines. Available LIBS data interpretation techniques have not presented a solution yet, so any method to be developed for automated on-board LIBS analysis would have to deal with both these effects. We believe that the artificial neural network-based advanced analytical method presented in this paper makes the first practical step on the way to realtime quantitative analysis of multiple elements of planetological interest. This study has used certified laboratory samples and rocks from meteorite-impact sites.

\section{LIBS EXPERIMENT}

The LIBS experimental setup used in the study is shown in Figure 1. It consists of a Q-switched Nd:YAG laser (Spectra Physics, LPY150, 1064 nm, 7 ns, 200mJ/pulse) operating at the repetition rate variable up to $20 \mathrm{~Hz}$. The laser energy is attenuated to $20 \mathrm{~mJ}$ with a combination of a $\lambda / 2$ wave plate and a polarizer. The laser beam is focused on the sample surface with a $75 \mathrm{~mm}$ focal-distance lens producing a spot of about $300 \mu \mathrm{m}$ in diameter. The plasma position is monitored with a CCD camera. In the experiments, the laser repetition rate was reduced to $1 \mathrm{~Hz}$ to allow the ablated dust to settle between laser pulses. LIBS spectra were measured in ambient air. A 7 $\mathrm{mm}$-focus, fused-silica collimator is used to focus the plasma emission into an optical fiber (solar-resistant - Ocean Optics, 2 $\mathrm{m}$ long, $600 \mu \mathrm{m}$ core diameter) connected to the spectrograph (50 cm Czerny-Turner spectrometer - Acton 500). The spec- 


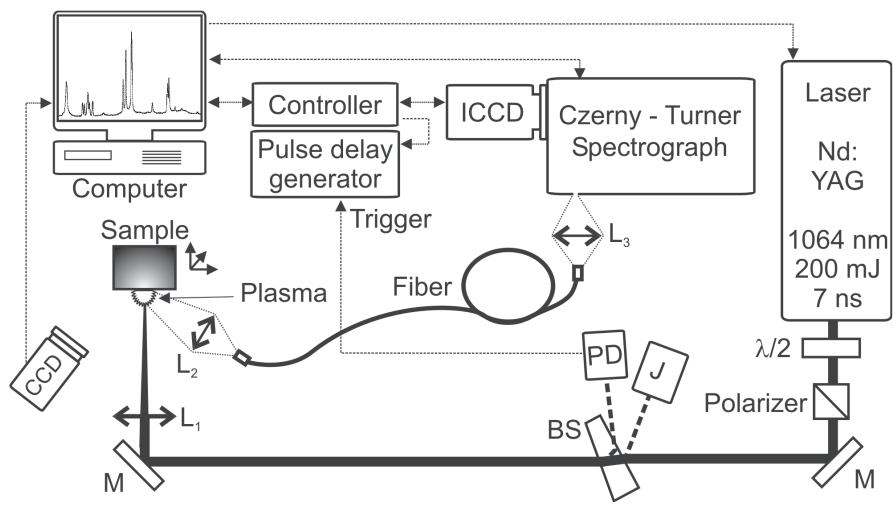

FIG. 1 Experimental LIBS setup. M - mirrors; BS - beam splitter; L1, L2, L3 - fused-silica lenses; $\lambda / 2$ - half-wave plate; PD - fast photodiode; J - Joule meter, CCD - camera used to monitor the plasma position.

trometer is equipped with a 600 lines $/ \mathrm{mm}$ grating providing spectral resolution of about $0.1 \mathrm{~nm}$ (entrance slit width of 25 $\mu \mathrm{m})$. The spectral range from 270 to $340 \mathrm{~nm}$ has been chosen to include characteristic peaks of $\mathrm{Fe}, \mathrm{Mg}, \mathrm{Mn}, \mathrm{Si}, \mathrm{Al}, \mathrm{Ca}$ and Ti. Spectra have been recorded using a gateable, intensified charge-coupled device (Princeton Instruments $256 \times 1024$ pixel ICCD array) cooled to $-35^{\circ} \mathrm{C}$. The intensifier gate delay and width were controlled with a pulse delay generator (PG200, Princeton Instruments). Gating parameters were chosen to ensure local thermal equilibrium conditions (the $0.5 \mu$ s delay and the $0.5 \mu \mathrm{s}$ gate width, [15]). The key system components are computer controlled with software, developed in the LabVIEW operational environment allowing for data averaging, storage, and the ANN real-time processing.

\section{ARTIFICIAL NEURAL NETWORKS FOR LIBS}

ANNs are now used in a broad range of areas such as pattern recognition, finances, data mining, battle scene analysis, etc. [16]. All these applications are such that it is practically impossible to build analytical or even numerical models taking into account all the many known - and often unknown - interconnected variables associated with the system to be analyzed. To our knowledge, there have been only two publications of the same authors exploring ANN as applied to quantitative LIBS analysis [17, 18]. Although this study was limited to measuring concentrations of only one element $(\mathrm{Cr})$ in soil, the ANN capability to determine accurate concentrations in different matrices was clearly demonstrated. The goal of our work has been development of an ANN processing tool capable of quantitative measurement of a large number of elements (ideally, all major as well as minor elements contained in most minerals of the exploration interest).

As illustrated in Figure 2, an artificial neuron identifies a weighted sum of inputs $x_{i}$, compares it to a given threshold (or a bias) $b$ and then transforms the resulting value into a response (output) $n$ using a nonlinear transfer function. The neurons are organized in layers to form a network. We have used a common three-layer network also called a perceptron (see Figure 2). Each neuron of the first (input) layer has a single input corresponding to the measured spectrum intensity
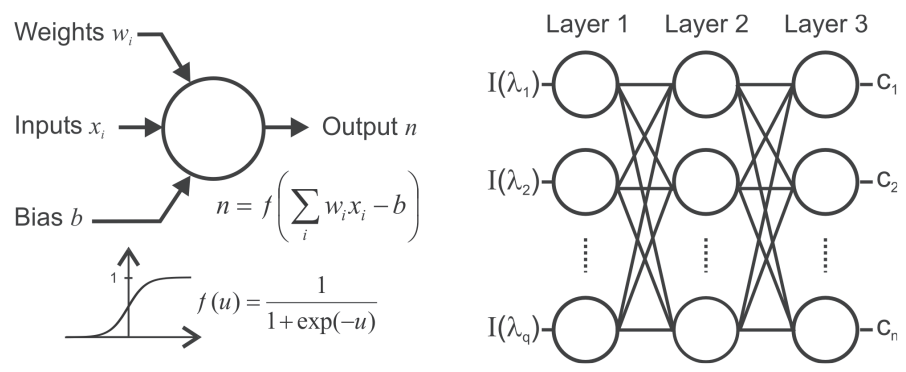

FIG. 2 Artificial neural network principle and architecture.

at one wavelength. The outputs of the last (output) layer constitute ANN results. Each neuron in this output layer is associated with one chemical element contributing to the spectrum. The number of neurons in the second layer (also called the hidden layer) is a free parameter. To produce accurate results, the algorithm needs to be trained (i.e., calibrated) with a set of reference spectra representative of the targets to be analyzed (in our case, rocks and soils). The training phase results in finding the best set of weights and bias values that would minimize the network output errors. This is done by using a backpropagation algorithm, which is based on a gradient descent allowing the network - after a certain number of iterations - to find the best fit to the training set of input-output pairs [16]. In order to reduce the number of iterations and hence the time needed for the training computation, we have used as inputs the intensities of lines corresponding to the emission lines of the elements of application interest (hereafter referred to as "reference lines"). We have selected one reference line per detectable element: Fe (274.95 nm), Mg (279.55 nm), Si (288.16 $\mathrm{nm}), \mathrm{Mn}(294.92 \mathrm{~nm}), \mathrm{Al}(309.27 \mathrm{~nm}), \mathrm{Ca}(317.93 \mathrm{~nm})$, and Ti (336.12 nm). These lines have been chosen based on following three criteria: (1) being a strong line in the spectral range probed; (2) no overlapping with other atomic lines, and (3) no significant self-absorption. Since the spectral range and resolution have been limited in our experiments, these three criteria have not been strictly satisfied for all the selected lines; nevertheless, a very satisfactory ANN performance has been achieved. Once the training phase was completed, a spectrum of unknown sample (i.e., intensities at selected wavelengths) was presented to the network, which quickly identified $(<$ $\mathrm{ms}$ ) the corresponding elemental composition.

\section{CALIBRATION AND VALIDATION OF THE ANN}

To train and validate the ANN algorithm we used LIBS spectra measured with certified powders of the following materials (by Brammer Standard Company, Inc; sample's number is shown in round brackets): andesite (JA-1), grey soil (2507), red soil (2501), black soil (2504), rocks (DC71306), sediments (SGHM-4), and basalt (JB-2). Table 1 shows the concentration range of the different constituents in the certified powders used and associated limit of detections, estimated for our setup. 


\begin{tabular}{|l|c|c|}
\hline Element & Concentration range $(\% \mathrm{w} / \mathrm{w})$ & LoD $(\% \mathrm{w} / \mathrm{w})$ \\
\hline $\mathrm{SiO}_{2}$ & $0.62-71.5$ & 0.13 \\
\hline $\mathrm{CaO}$ & $0.17-30.0$ & 0.01 \\
\hline $\mathrm{Al}_{2} \mathrm{O}_{3}$ & $0.10-15.2$ & 0.03 \\
\hline $\mathrm{Ti}_{2} \mathrm{O}_{3}$ & $0.02-1.56$ & 0.002 \\
\hline $\mathrm{MgO}$ & $0.48-21.8$ & 0.001 \\
\hline $\mathrm{MnO}$ & $0.01-0.22$ & 0.01 \\
\hline $\mathrm{Fe}_{\text {total }}$ & $0.21-14.3$ & 0.01 \\
\hline
\end{tabular}

TABLE 1 Concentration range in the calibration set samples and associated Limit of Detection (LOD).

Each sample was compacted in a stainless steel pellet under a pressure of about 10 tons $/ \mathrm{cm}^{2}$. Two sets of 50 spectra $(5 \mathrm{spec}-$ tra across the sample surface and 10 at different depths) were recorded for each sample; the first (calibration) set was used for the algorithm training. The network performance test was carried out with the second (validation) set. The background was subtracted from each spectrum. Reference-line intensities were retrieved with a common spectroscopic peak-detection algorithm and then normalized on the $274.95 \mathrm{~nm}$ iron $(\mathrm{Fe})$ line intensity. These normalized intensities were then averaged for both sets of 50 spectra, and the mean values were used for training and validation. The iron line was chosen for normalization because Fe is present (often as a major constituent) in most natural rocks; similar results (not reported here) have been obtained using a silicon (Si) line at $288.16 \mathrm{~nm}$.

As our detection wavelength range did not allow measurements of all possible rock-forming elements (in particular $\mathrm{Na}$, $\mathrm{K}, \mathrm{C}$ and $\mathrm{P}$ ), the network was trained with the ratios $r_{X}=$ $c_{X} / \sum_{i=1}^{n} c_{i}$. Each ratio represents the ratio of the element $X$ concentration and the sum of concentrations of all the detectable elements ( $n=7$ in our case). Thus, ANN processing gives $n$ values of $r_{X}$ corresponding to the $n$ measured elements. Assuming that all the major elements composing the sample are detected, the sum $\sum_{i=1}^{n} c_{i} \approx 1$ and the ratios $r_{X}$ are equal to element concentrations $c_{X}$. Besides, since no oxygen line was detected, ratios were calculated with known concentrations of the elements in their principal oxide forms $\mathrm{MgO}, \mathrm{SiO}_{2}, \mathrm{MnO}, \mathrm{Al}_{2} \mathrm{O}_{3}, \mathrm{CaO}, \mathrm{TiO}_{2}$ and $\mathrm{Fe}_{\text {total }}$, the latter being defined as out of $\mathrm{Fe}_{2} \mathrm{O}_{3}+\mathrm{FeO}$. The algorithm's free parameters were optimized with a step-by-step approach described in [18], which minimizes the deviation between the ANN concentration results and the certified values. A relative deviation of about $8 \%$ averaged over all the validation set of samples has been obtained, demonstrating excellent performance of the network despite the fact that elemental concentrations were measured in different matrices.

\section{APPLICATION TO NATURAL ROCKS}

To demonstrate that the ANN processing would meet fieldmeasurement requirements, we also studied four different natural rocks taken from a recently identified meteorite impact site in Egypt [19]: the impact glass (DAK-20 and DAK54), sandstone (DAK-34), and volcanic glass (DAK-44). These samples constitute mixtures of rocks and soil (regolith) making it a nearly ideal analog of the Martian surface material.
The selected rocks had an amount of $\mathrm{SiO}_{2}$ representative of the set of certified samples with low $\mathrm{Na}, \mathrm{P}, \mathrm{C}$ and $\mathrm{K}$ concentrations. The ANN parameters and the LIBS experimental conditions for these rocks were the same as for the certified powders. We used the calibration (training) of ANN LIBS done on powders to analyse these four a priori unknown natural rocks. To reduce measurement dispersion due to the rock heterogeneity, we used a larger surface sampling (10 spectra across the sample surface and 5 at different depths). Normalized LIBS spectra of the studied rocks are shown in Figure 3, where the used reference lines are also presented. The standard deviation for the obtained spectra depended on the line and was within $5 \%$ for $\mathrm{Si}, \mathrm{Ca}, \mathrm{Al}, \mathrm{Ti}, \mathrm{Mg}, 7 \%$ for $\mathrm{Fe}$ and $10 \%$ for $\mathrm{Mn}$.

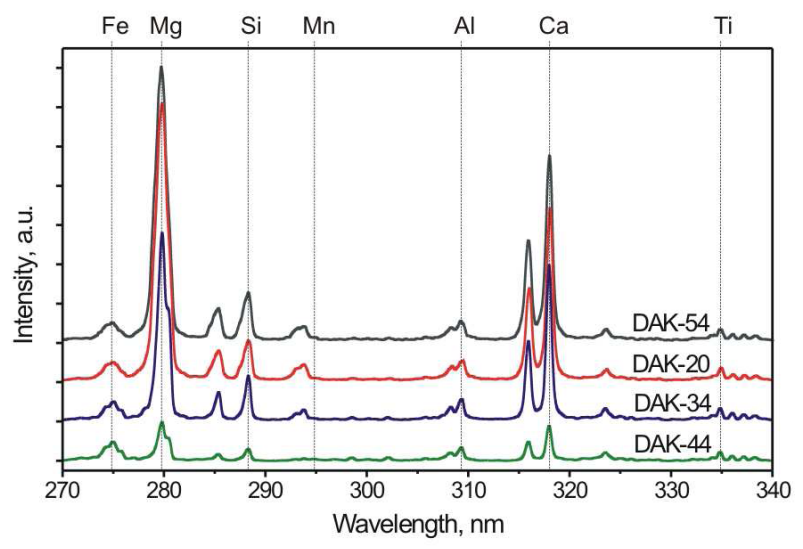

FIG. 3 LIBS spectra of natural rocks normalized on the intensity of the $274.95 \mathrm{~nm}$ iron line. Plots have been shifted for better observation.

The ANN-determined concentrations for these rocks and associated standard uncertainties are summarized in Table 2. The uncertainties were obtained with a Student factor equal to $t=2.571$ (confidence level of 95\%). We used the formula $t \times s / \sqrt{n}$ with $n$ the number of replicates $(n=50)$ and $s$ the standard deviation of the ANN results. The uncertainty of the concentrations given by the ANN algorithm was in the range of $5 \%$ for all measured elements. To make a comparison, concentrations of each main element in the entire rock volume were also retrieved with bulk XRF (X-Ray Fluorescence) measurements. The mean deviation of LIBS results from certified XRF data was $15 \%$ demonstrating, as for the validation set, a very good ANN performance for different rock matrices. From all the ANN-determined concentrations, the best result has been obtained for $\mathrm{SiO}_{2}$ at about $4 \%$ deviation (averaged and $10 \%$ worst-case). The results for $\mathrm{MgO}$ and $\mathrm{CaO}$ have also been quite accurate $(<15 \%$ deviation) considering that the $\mathrm{Mg}$ and $\mathrm{Ca}$ lines are self-absorbed at concentrations higher than $\sim 2 \%$ and $\sim 8 \%$, respectively. Similar results have been obtained for $\mathrm{Al}_{2} \mathrm{O}_{3}$ and $\mathrm{Fe}_{\text {total }}$ (deviations of $\sim 20 \%$ ) that may be explained by the heterogeneity of the studied samples [19] and spectral overlapping of the $\mathrm{Al}, \mathrm{Fe}$ and Ti lines. Acceptable deviation was obtained for the MnO sample ( 25\%) in which elemental concentrations were low. Figure 4 illustrates graphically the results of Table 1 by plotting the ANN retrieved concentrations against the XRF concentrations. This Figure demonstrates the algorithm capability to retrieve the 


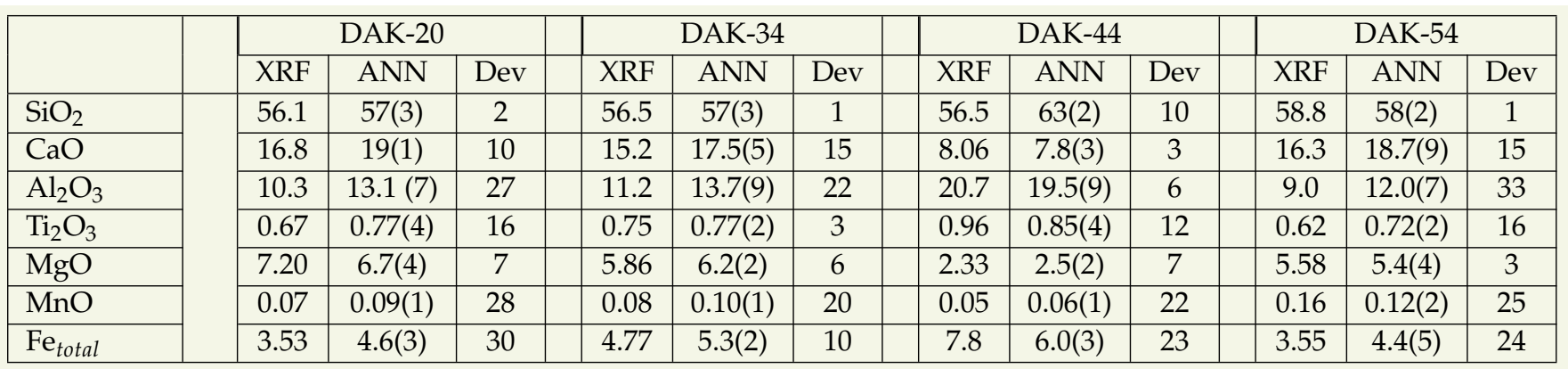

TABLE 2 Natural rock measurements. Comparison of the XRF and LIBS ANN measured concentrations (weight \%). Dev: deviation from XRF in \% [(XRF-ANN)/XRF]. Numbers in parentheses represent the standard uncertainty in units of the least significant digit.

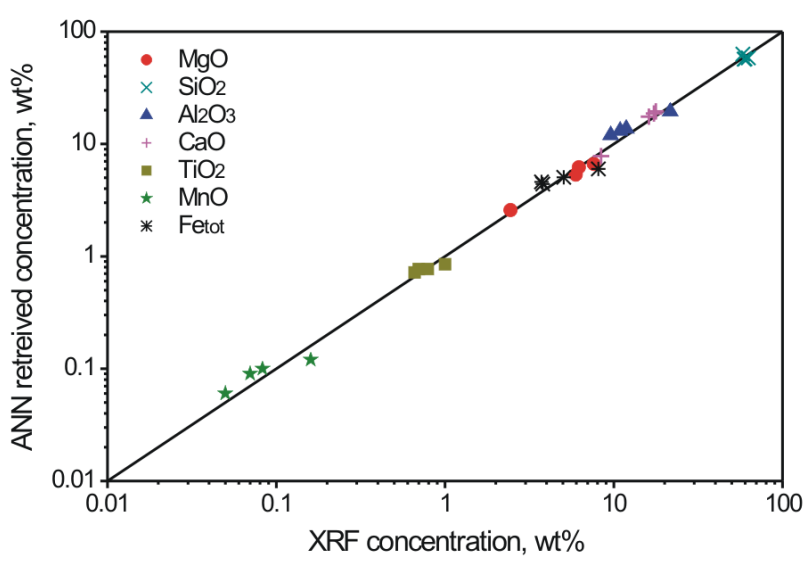

FIG. 4 Comparison between the concentrations retrieved by the ANN from LIBS measurements and the XRF concentrations of $\mathrm{MgO}, \mathrm{SiO}_{2}, \mathrm{MnO}, \mathrm{Al}_{2} \mathrm{O}_{3}, \mathrm{CaO}, \mathrm{TiO}_{2}$ and $\mathrm{Fe}_{\text {total }}$.

different element concentrations in very large dynamic range, up to 4 orders of magnitude.

In short, these results show excellent processing capability of the ANN as applied to different mineral matrices, despite spectral overlapping of lines and self-absorption. Noticeably, the ANN processing accuracy for LIBS of rocks is naturally limited by the heterogeneity of the samples. In addition, concentrations of some elements present in the rocks - such as $\mathrm{Na}, \mathrm{K}, \mathrm{P}$ and $\mathrm{H}$ - have not been measured. This induces a prediction bias (about $4 \%$ of the undetected elements for any of the studied rocks). Therefore, the deviations obtained for the studied rock samples can be considered as being close to the validation set values (obtained with powders). Using a broader UV-visible-IR spectral range (e.g. 200-800 nm with Echelle-type spectrometer) would certainly improve the ANN accuracies by allowing better satisfying reference line selection criteria (see Section 3). A broader spectral range would also further increase the ANN versatility by enabling measurements of all major elements (including $\mathrm{Si}, \mathrm{Ca}, \mathrm{O}, \mathrm{Ti}, \mathrm{Fe}$, $\mathrm{Al}, \mathrm{Mg}, \mathrm{Mn}, \mathrm{Na}, \mathrm{K}, \mathrm{H}, \mathrm{C}$ and $\mathrm{P}$, which comprise the majority of most natural rocks).

Compared to the calibration-free approach [20], recently demonstrated for quantitative multi-elemental analysis of rocks, ANN processing performs much better [21, 22]. It can be estimated that the measurement accuracies achieved in $[21,22]$ have been of $30 \%$ (or worse) for all almost all the major and minor rock constituents except for Si $(<15 \%)$. However, careful evaluation requires similar experimental conditions and instrumentation to be used. A direct comparison of these two approaches using a larger set of rock samples would make an interesting subject of further studies.

\section{CONCLUSION}

We have described a processing technique based on a neural network algorithm, which allows automated quantitative analysis of LIBS spectra. Following adequate calibration and validation, this processing has been successfully applied to analyses of different silicate rocks. Simultaneous concentration measurements of a group of seven elements $(\mathrm{Fe}, \mathrm{Mg}, \mathrm{Si}$, $\mathrm{Mn}, \mathrm{Al}, \mathrm{Ca}$ and $\mathrm{Ti}$ ) have been demonstrated. A mean deviation better than $15 \%$ from XRF-measured values has been achieved. In particular, ANN has shown an excellent potential to account for the effects of matrices and line spectral overlapping in a large range of concentrations and line intensities. The procedure simplicity and the fast ANN response make this processing a promising tool for fully automated, real-time LIBS analysis in such applications as rock elemental screening and the target selection for other in-situ analyses in planetary landing missions, Earth geology, natural-resource exploration, mining, etc.

\section{ACKNOWLEDGEMENTS}

The authors are grateful to M. Sabsabi, S. Laville, J. Lucas, and D. Gratton for fruitful discussions, A. Hamel for help in experiments, and the Space Technology Research and Development Program (STRP) of the Canadian Space Agency for financial support.

\section{References}

[1] D. A. Cremers and L. J. Radziemski, Handbook of laser-induced breakdown spectroscopy (Wiley, 2006).

[2] A. Ciucci, V. Palleschi, S. Rastelli, R. Barbini, F. Colao, R. Fantoni, A. Palucci, S. Ribezzo and H. J. L. van der Steen, "Trace pollutants analysis in soil by a time-resolved laser-induced breakdown spectroscopy technique" Appl. Phys. B 63, 185 (1996).

[3] J. Gruber, J. Heitz, H. Strasser, D. Bauerle and N. Ramaseder, "Rapid in-situ analysis of liquid steel by laser-induced breakdown" Spectrochim. Acta B 56, 685 (2001). 
[4] L. St-Onge, E. Kwong, M. Sabsabi and E. B. Vadas, "Quantitative analysis of pharmaceutical products by laser-induced breakdown spectroscopy" Spectrochim. Acta B 57, 1131 (2002).

[5] M. Z. Martin, S. D. Wullschleger, C. T. Garten, Jr., and A. V. Palumbo, "Laser-induced breakdown spectroscopy for the environmental determination of total carbon and nitrogen in soils" Appl. Optics 42, 2072 (2003).

[6] S. Morel, N. Leone, P. Adam, and J. Amouroux, "Detection of Bacteria by Time-Resolved Laser-Induced Breakdown Spectroscopy" Appl. Optics 42, 6184 (2003).

[7] A. K. Knight, N. L. Scherbarth, D. A. Cremers and M. J. Ferris, “Characterization of Laser-Induced Breakdown Spectroscopy (LIBS) for Application to Space Exploration" Appl. Spectrosc. 54, 331 (2000).

[8] R. C. Wiens, R. E. Arvidson, D. A. Cremers, M. J. Ferris, J. D. Blacic, F. P. Seelos IV and K. S. Deal, "Combined remote mineralogical and elemental identification from rovers - Field and laboratory tests using reflectance and laser-induced breakdown spectroscopy" J. Geophys. Res. 107, 3 (2002).

[9] B. C. Clark III, A. J. Castro, C. D. Rowe, A. K. Baird, H. J. Rose Jr., P. Toulmin III, R. P. Christian, W. C. Kelliher, K. Keil and G. R. Huss, "The Viking $X$ ray fluorescence experiment - Analytical methods and early results" J. Geophys. Res. 82, 4577 (1977).

[10] R. Rieder, T. Economou, H. Wanke, A. Turkevich, J. Crisp, J. Breckner, G. Dreibus and H. Y. McSween Jr, "The Chemical Composition of Martian Soil and Rocks Returned by the Mobile Alpha Proton $X$-ray Spectrometer: Preliminary Results from the X-ray Mode" Science 278, 1771 (1997).

[11] S. Maurice, R. Wiens, G. Manhès, D. Cremers, B. Barraclough, J. Bernardin, M. Bouyé, A. Cros, B. Dubois, E. Durand, S. Hahn, D. Kouach, J.-L. Lacour, D. Landis, T. Moore, L. Parès, J. Platzer, M. Saccoccio, B. Sallé and R. Whitaker, "ChemCam Instrument for the Mars Science Laboratory (MSL) Rover" 36th Annual Lunar and Planetary Science Conference 1735, (2005).

[12] G. Bazalgette Courrèges-Lacoste, B. Ahlers and F. Rull Pérez, “Combined Raman spectrometer/laser-induced breakdown spectrometer for the next ESA mission to Mars" Spectrochim. Acta A, in press.

[13] R. S. Harmon, F. C. DeLucia; C. E. McManus, N. J. McMillan, T. F.
Jenkins, M. E. Walsh and A. Miziolek, "Laser-induced breakdown spectroscopy - An emerging chemical sensor technology for realtime field-portable, geochemical, mineralogical, and environmental applications" Appl. Geochem. 21, 730 (2006).

[14] A. S. Eppler, A. D. Cremers, D. D. Hickmott, M. J Ferris and A. C. Koskelo, "Matrix effects in the detection of $\mathrm{Pb}$ and $\mathrm{Ba}$ in soils using laser-induced breakdown spectroscopy" Appl. Spectrosc. 50, 1175 (1996).

[15] F. Colao, R. Fantoni, V. Lazic and A. Paolini, “LIBS application for analyses of martian crust analogues: search for the optimal experimental parameters in air and $\mathrm{CO}_{2}$ atmosphere" Appl. Phys. A 79, 143 (2004).

[16] M. T. Hagan, H. B. Demuth and M. B. Beale, Neural Network Design, Boston, Mass (PWS Publishing, 1996).

[17] J.-B. Sirven, B. Bousquet, L. Canioni, L. Sarger, S. Tellier, M. PotinGautier and I. Le Hecho, "Qualitative and quantitative investigation of chromium-polluted soils by laser-induced breakdown spectroscopy combined with neural networks analysis" Anal. Bioanal. Chem. 385, 256 (2006).

[18] J. B. Sirven, B. Bousquet, L. Canioni and L. Sarger, "Laser-induced breakdown spectroscopy of composite samples: Comparison of advanced chemometrics methods" Anal. Chem. 78, 1462 (2006).

[19] G. R. Osinski, H. P. Schwarcz, J. Smith, M. R. Kleindienst, A. F. C. Haldemann, and C. S. Churche, "Evidence for a 100-200 ka meteorite impact in Western Egypt" Earth Planet. Sc. Lett. 253, 378 (2007).

[20] A. Ciucci, M. Corsi, V. Palleschi, S. Rastelli, A. Salvetti, and E. Tognoni, "New Procedure for Quantitative Elemental Analysis by Laser-Induced Plasma Spectroscopy" Appl. Phys. A, 53, 960 (1999).

[21] B. Sallé, J.-L. Lacour, P. Mauchien, P. Fichet, S. Maurice, and G. Manhès, "Comparative study of different methodologies for quantitative rock analysis by Laser-Induced Breakdown Spectroscopy in a simulated Martian atmosphere" Spectrochim. Acta B 61, 301 (2006).

[22] F. Colao, R. Fantoni, V. Lazic, A. Paolini, F. Fabbri, G. G. Ori, L. Marinangeli and A.Baliva, "Investigation of LIBS feasibility for in situ planetary exploration: An analysis on Martian rock analogues" Planet. Space Sci. 52, 117 (2004). 